\title{
A survey-based assessment of how existing and potential electric vehicle owners perceive range anxiety
}

\author{
Dario Pevec a, * , Jurica Babic a , Arthur Carvalho ${ }^{\mathrm{b}}$, Yashar Ghiassi-Farrokhfal ${ }^{\mathrm{c}}$, \\ Wolfgang Ketter ${ }^{c, d}$, Vedran Podobnik ${ }^{a}$ \\ a University of Zagreb, Croatia \\ ${ }^{\mathrm{b}}$ Miami University, USA \\ c Erasmus University, Netherlands \\ ${ }^{\mathrm{d}}$ University of Cologne, Germany
}

\section{A R T I C L E I N F O}

\section{Article history:}

Received 15 December 2019

Received in revised form

25 April 2020

Accepted 10 June 2020

Available online 24 July 2020

Handling editor: Cecilia Maria Villas Bôas de Almeida

\section{Keywords:}

Range anxiety

Electric vehicles

Driving range

Charging stations

\begin{abstract}
A B S T R A C T
Electric vehicle (EV) owners enjoy many positive aspects when driving their cars, including low running costs and zero tailpipe gas emissions, which makes EVs a clean technology provided that they are sourced through renewable sources, e.g., biomass, solar power, or wind energy. However, their driving behaviour is often negatively affected by the so-called range anxiety phenomenon, i.e., a concern that an EV might not have enough driving range to reach the desired destination due to its limited battery size. The perception of range anxiety may also affect potential buyers in their decisions on whether to purchase an internal combustion engine vehicle as opposed to an EV. This paper investigates some factors that influence range anxiety through a comparative analysis of two target groups: (i) existing EV owners, and (ii) non-EV owners (i.e., potential EV owners). The specially crafted survey was used to collect range anxiety data from more than 200 participants. In particular, participants provided their perceptions on (i) the potential relationship between existing gas station infrastructure and the desired EV charging station infrastructure, and (ii) the potential relationship between range anxiety and two influencing variables, namely the current state of charge and remaining range. Concerning the existing gas station infrastructure, evidence suggests that both target groups think that the distances between gas stations could be increased. Moreover, our analysis shows that the desired distances between charging stations correspond to the distances between the existing gas stations, which indicates that both EV owners and non-EV owners have a common view on the optimal gas station and charging station topology. Furthermore, we find that the type of settlement (urban vs rural) influences preferred distances, where both target groups living in cities desire shorter distances, and that non-EV owners, as opposed to EV owners, are more prone to be affected by the state of charge and remaining range. Quantitatively, we are able to define a measure for range anxiety, which is connected with the preferred distance between two neighbouring charging stations. Throughout our analyses, we find that the mean preferred distance between two neighbouring charging stations is $7 \mathrm{~km}$, but this value significantly differs based on the settlement type of a (potential) EV owner.
\end{abstract}

(c) 2020 Elsevier Ltd. All rights reserved.

\section{Introduction}

Various environmental concerns, from climate-related changes

\footnotetext{
* Corresponding author.

E-mail addresses: dario.pevec@fer.hr (D. Pevec), jurica.babic@fer.hr (J. Babic), arthur.carvalho@miamioh.edu (A. Carvalho), y.ghiassi@rsm.nl (Y. GhiassiFarrokhfal), ketter@wiso.uni-koeln.de (W. Ketter), vedran.podobnik@fer.hr (V. Podobnik).
}

and illnesses to rising seas, are considered to be among the most prominent challenges humans face (Quinn et al., 2016; Paavola, 2017). One of the prime factors behind numerous environmental problems is air pollution, of which the transportation domain is a major contributor to the $\mathrm{CO}_{2}$ footprint. For example, according to Van Fan et al. (2018), more than 25\% of the total greenhouse emissions in Europe are attributed to the transportation sector. Reacting to the global increase of the number of personal vehicles (Agency, 2019), academia, industry, and governments are putting 
effort into tackling environmental concerns by inventing and supporting alternative transportation technologies, commonly known as alternative fuel vehicles (AFV). AFVs can be fueled by electricity, hydrogen, biodiesel or solar power (Valogianni et al., 2018, 2020; Kahlen et al., 2018; Akakpo et al., 2019). In our paper, we focus on electric vehicles (EVs), a subgroup of AFVs that are fueled by electricity. Ketter et al. (2016) argued that EVs can significantly lower the transportation sector's negative impact on greenhouse gas footprint, especially if renewable sources are significantly present in their production.

We note that more than 1 million EVs were sold in 2017, representing a $50 \%$ growth when compared to 2016, and leading to more than 3 million electric vehicles on the roads globally (Bunsen et al., 2018). There are two main driving factors behind this accelerated adoption of EVs. First, academia and industry managed to find ways to produce batteries of greater capacities at lower prices, resulting in increased EV autonomy and longer driving ranges. Second, governments have been providing incentives for purchasing and operating EVs, such as lower registrations costs and free public charging (Bjerkan et al., 2016).

Nonetheless, EVs are still far away from mass adoption. For example, EVs correspond to more than $1 \%$ of the market share among private vehicles in only three countries globally, namely Norway, Sweden, and China (Statista, 2018). The range anxiety phenomenon, i.e., an EV driver's fear of running out of electricity before reaching another available charging station (Neubauer and Wood, 2014), is one of the most important factors that influence new-vehicle buyers when deciding on whether to purchase a traditional internal combustion engine (ICE) vehicle as opposed to an EV (Adnan et al., 2017). We note that range anxiety can be lowered by either increasing EV autonomy or by enhancing the existing charging infrastructure.

This paper focuses on the charging infrastructure aspect by assessing variables that impact range anxiety. Our first overarching research question is: "How do existing EV owners, as well as potential EV owners, perceive charging station infrastructure in comparison to the existing gas station infrastructure, considering the distance between two neighbouring chargers and gas stations?". Answering this question enables us to understand both the relationship between the degree of charging station infrastructure development and range anxiety as well as to make connections with the gas station infrastructure, which is significantly more mature when compared to charging station networks. Our second overarching research question is: "To what extent do different key EV parameters influence the range anxiety of potential EV owners, and how does that compare to existing EV owners?". Answering this question enables us to understand the relationship between the state of charge (SoC), i.e., the remaining capacity of an EV battery, and the EV driver's decision to charge or not the vehicle. Furthermore, the second research question also enables us to understand whether SoC and/ or the driving range, i.e., the remaining range the $\mathrm{EV}$ can reach, have a significant influence on range anxiety. Both questions are answered by analyzing data collected through a specially designed survey aimed at both existing and potential EV owners, i.e., drivers who do not own an EV. In total, we collected responses from more than 200 participants. The survey consisted of three parts: (i) demographic questions; (ii) a questionnaire comparing charging station and gas station infrastructure; and (iii) 5 arbitrarily generated scenarios through which survey respondents gave opinions about their willingness to charge. This enabled us to categorize survey respondents based on different individual characteristics (e.g. age), contextual information (e.g., settlement type), and EVrelated parameters (e.g., EV ownership).

The above said, the main objective of this paper is to quantify range anxiety, which we connect with the desired distance between neighbouring charging stations, thus filling a gap in the current literature on EVs. Furthermore, we provide a comparison of EV owners' and non-EV owners' perceptions of factors that drive range anxiety. Such a comparison is of paramount importance because it has been assumed that potential EV owners, being perhaps less knowledgeable about EVs, manifest a higher degree of range anxiety (Plötz et al., 2014). Finally, we study how the hierarchy of settlements influences the manifested range anxiety. We note that this paper is an extension of the research by Pevec et al. (2019), where only the perspective of potential EV owners was taken into account, and the work by Pevec et al. (2018), where range anxiety was derived from the mean driving speed in cities and from how far ICE vehicle owners are willing to travel to reach another gas station.

\section{Related work}

In this section, we review the most relevant related studies from the perspective of the research methodology (i.e., survey) and the research domain (i.e., range anxiety). Starting with the former, as a consequence of the scarcity of publicly available real-life EV-related data, the research methodologies employed in this field often rely on alternative means of data collection. In particular, common approaches include simulations, draws from various probability distributions, and surveys, which is the methodology we use in our research. The survey-based approach has been commonly used when assessing (potential) EV owners' willingness to pay for the charging service, as presented by Babic et al. (2017) and Dorcec et al. (2019). Similar to our approach, those authors created EVrelated scenarios to collect data from a diverse set of participants. Moreover, although the underlying question was always the same (i.e., how much is a participant willing to pay for the charging service?), the authors varied some parameters, such as the time of the day, SoC, and remaining EV range, so as to create different scenarios. Besides assessing willingness to pay, Jensen et al. (2016) and Lebeau et al. (2012) used a survey-based methodology to forecast EV market penetration, while Higgins et al. (2012) and Ko and Hahn (2013) used surveys to determine factors that influence potential consumers to purchase or not an EV.

Regarding range anxiety, the work by Rauh et al. (2015) was aimed towards assessing how range anxiety manifests in EV owners in comparison to potential EV owners. The test comprised of driving a provided EV with a predetermined SoC. It was concluded that long-term EV owners are less affected by the range anxiety phenomenon than those participants with no or very little experience with EVs. Furthermore, Rauh et al. (2015) concluded that SoC can influence range anxiety. However, it was still unclear whether and how range anxiety could be mitigated. Filling the gap, the studies by King et al. (2015) and Bonges and Lusk (2016) were primarily focused on the challenges of mitigating range anxiety. To do so, they proposed a novel model for EV usage, namely "EVs on demand". After modelling the pricing scheme of the proposed EV usage model, those authors concluded that the proposed model is more economically acceptable comparing to the all the subsidies that governments offer for the purchase of EVs.

Unlike the previously described research, the studies by Jung et al. (2015) and Franke and Krems (2013) were aimed towards explaining how the experience of owning an EV influences range anxiety. Jung et al. (2015) recruited 73 drivers to drive an EV for 19 miles. The authors manipulated parameters such as the $\mathrm{SoC}$ in the beginning of a drive and the clarity of the EV user interface. Jung et al. (2015) concluded that the lower the SoC and the clarity of the user influence are, the more pronounced range anxiety is. Franke and Krems (2013) also used test drivers without previous experience with electric vehicles in their experiments. All 
participants were provided with an EV for three months in order to assess their range anxiety over time. Franke and Krems (2013) concluded that range anxiety significantly decreased with time. This is a clear conclusion that owning an EV lessen the range anxiety a driver experiences. While the above research was based on how to alleviate range anxiety issues, the work by Neubauer and Wood (2014) was rather concentrated on studying the consequences of range anxiety on EV lifetime utility. The authors used computer simulations based on one-year-long trip data in order to model driving behavior (i.e., human factor). The results of the simulations show that the drivers affected by range anxiety significantly diminish the utility factors of their EVs. Moreover, range anxiety becomes lower and, therefore, the lifetime utility of EVs increase with developed charging infrastructure.

Besides the studies that focus specifically on range anxiety, related work also includes broader research areas that deal with EV owners' charging behavior and alternative charging methods, such as smart charging based on the grid load during peak times, which in turn was systematically investigated in the studies by Valogianni et al. (2012), Valogianni and Ketter (2016), and Kahlen et al. (2018). Finally, the work by Noel et al. (2019) addressed the range anxiety phenomenon from the psychological and societal aspect, rather than technical. The authors based their research on the "rhetoric of reaction" framework (Hirschman, 1991), through which any action performed to tackle a specific challenge falls into one of three possible categories: (i) perversity; (ii) futility; or (iii) jeopardy. Noel et al. (2019) performed more than 200 semi-structured interviews as well as a survey with more than 5,000 participants to assess range anxiety among the population across 17 Nordic cities. The authors concluded that the answers from a significant number of participants are in agreements with the underlying framework. For example, some of the participants stated that EVs have a high chance to catch fire, while ignoring the fact that ICE vehicles display an even greater chance of the same, thus confirming the jeopardy thesis. Perversity and futility were demonstrated through the fact that a significant number of participants stated that EVs have a negative impact on the environment, even though these cars are designed with the goal of reducing harmful gas emissions and there are numerous studies that confirm this fact. Therefore, the research conclusion by Noel et al. (2019) was that range anxiety should be considered as a rhetorical construction that is neither technical nor psychological. As such, the solution to the range anxiety issue does not necessarily lie in the development of the charging infrastructure. Instead, it is about socio-technical benefits, such as free tolls, parking, charging, and other advantages that can potentially influence a decision to purchase an EV and, thus, lower range anxiety.

Based on our literature review, it is evident that the range anxiety phenomenon presents a significant challenge in the EV research domain. Moreover, range anxiety has been studied from different perspectives by different research communities. Unlike the related studies presented in this section, our goal in this paper is to quantitatively measure range anxiety by surveying both owners and non-owners of EVs. To achieve this goal, our work connects range anxiety with the desired distance between neighbouring charging stations. Another contribution of our work is on the analysis of the charging infrastructure in comparison to the well-developed gas station infrastructure.

\section{Research methodology}

This section describes the methodology we followed in our research. In particular, we rely on the design of well-crafted surveys (Couper et al., 2001) and follow the cross-industry standard process for data mining (CRISP-DM) methodology (Wirth and Hipp, 2000). The end-to-end methodology is depicted in Fig. 1 and described in

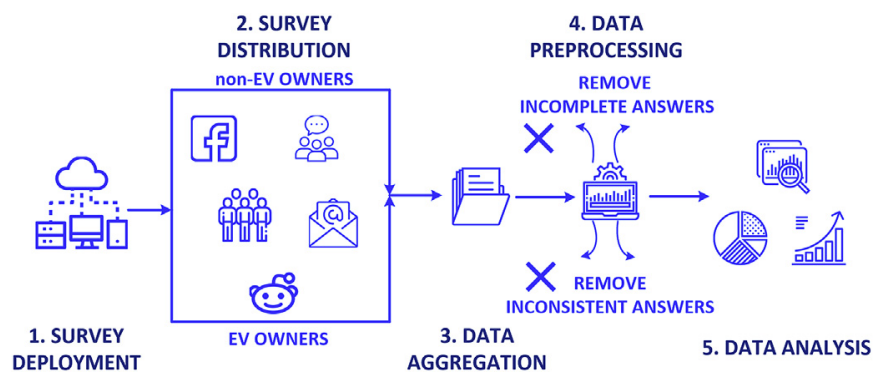

Fig. 1. Research methodology.

the following paragraphs alongside with a detailed description of the survey design.

\subsection{Data flow}

Our survey was developed using the LimeSurvey tool, which can be used both as a service or deployed on one's private infrastructure. We decided to use the latter approach for survey deployment, the first step in Fig. 1, since we needed access to the tool's source code as well as the flexibility in the access control mechanisms and better integration with our established Web domain.

The second step of the methodology, survey distribution, was to distribute the aforementioned survey among a diverse set of participants. In particular, We distributed the survey targeting two different populations: (i) non-EV owners; and (ii) EV owners. To better cover the non-EV population, we used various communication channels to maximize the reach and achieve heterogeneity among survey respondents in terms of demographics (e.g., age, gender, income, settlement hierarchies considering population density, or knowledge about EVs). More specifically, we used Facebook, various forums, Reddit, and a word-of-mouth approach. For the group of participants who are EV owners, besides the previous communication channels, we also relied on specialized EVrelated forums, companies that provide electricity and work on charging station infrastructure development, such as HEP ${ }^{1}$ Croatian electricity provider, and Facebook groups containing EV owners from all over the world. Our approach for survey distribution ensures that we have enough participants, both EV owners and potential EV owners, i.e., drivers that still do not own an EV. A detailed statistical analysis about the survey participants is described in Section 4.

The next step of the methodology, data aggregation, includes the aggregation of the responses into a single dataset that is appropriate for further analysis, as well as the grouping of the participants into one of the studied groups based on EV ownership. The data aggregation phase is followed by the data pre-processing phase, where answers that are incomplete (e.g., when a participant left the survey before finishing it), as well as inconsistent answers (e.g., effortless answers where all the reported values are the same) were removed. Besides removing uninformative responses, we transformed the variables in order to eventually perform a statistical analysis. In other words, our data pre-processing step ensures the availability of a high-quality dataset in the last phase of our methodology, namely the data analysis step.

\subsection{Survey design}

The survey consists of the following parts: (i) demographic

\footnotetext{
${ }^{1}$ https://www.hep.hr/.
} 
Table 1

Set of demographic questions with potential answers and preconditions.

\begin{tabular}{|c|c|c|c|}
\hline \# & QUESTION & ANSWERS & CONDITION \\
\hline 1 & What is your gender? & (“Male"; “Female") & None \\
\hline 2 & What is your country? & List of all countries & None \\
\hline 3 & What is your age? & Number between 1 and 100 & None \\
\hline 4 & What is your working status? & ("Student"; “Employed"; “Unemployed"; “Retired") & None \\
\hline 5 & What is your annual net income? (\$) & Number & None \\
\hline 6 & Do you have a driving licence? & ("Yes"; “No") & None \\
\hline 7 & Please evaluate your familiarity with the concept of electric vehicles & $\begin{array}{l}\text { ("Never heard of it"; "Heard of it, but I am not familiar"; "I know } \\
\text { something"; "I am very familiar") }\end{array}$ & None \\
\hline 8 & How many cars have you owned so far? & Number & None \\
\hline 9 & $\begin{array}{l}\text { In your opinion, what should be the maximal distance between two charging } \\
\text { stations in a city (in } \mathrm{km}, 1 \mathrm{~km}=0,62 \text { miles)? }\end{array}$ & Number & None \\
\hline 10 & Do you own a car now? & (“Yes"; “No") & None \\
\hline 11 & How many vehicles do you have in your household? & Number & $\begin{array}{l}\text { If Question } \\
\# 10=\text { "YES" }\end{array}$ \\
\hline 12 & Do you own or have an EV? & ("Yes"; “No") & $\begin{array}{l}\text { If Question } \\
\# 10=\text { "YES" }\end{array}$ \\
\hline 13 & What model is your EV (e.g., Nissan Leaf)? & Open text & $\begin{array}{l}\text { If Question } \\
\# 12=\text { "YES" }\end{array}$ \\
\hline 14 & What is the capacity of your EV battery (kWh)? & Number & $\begin{array}{l}\text { If Question } \\
\# 12=\text { "YES" }\end{array}$ \\
\hline 15 & $\begin{array}{l}\text { At what state of charge (remaining battery) do you usually charge your EV? (In } \\
\text { percentage) }\end{array}$ & Number & $\begin{array}{l}\text { If Question } \\
\# 12=\text { "YES" }\end{array}$ \\
\hline
\end{tabular}

questions; (ii) a questionnaire comparing charging station and gas station infrastructure; and (iii) 5 arbitrarily generated scenarios through which survey respondents give opinions about their willingness to charge. All questions were asked in English.

The first group of questions presented to the participants concerns demographics. The first part of this question group contains rather standard questions (see the first five questions in Table 1), e.g., gender, country, age, and income. Answering all questions was not mandatory, e.g., respondents did not have to report their annual income. The second part of the demographic question group (questions 6 to 11 in Table 1 ) is oriented towards assessing the participants ability to drive as well as to better understand what type of vehicle each participant owns. Lastly, the third group of questions in this category (questions 12 to 15 in Table 1) are domain specific and they serve to separate EV owners from non-EV owners. We used the technique of hidden questions, meaning that, for example, questions 13 through 15 , which are EV-related questions, are not be visible to someone who does not own an EV.

The second group of questions is about participants' preferences regarding the development of $\mathrm{EV}$ charging infrastructure and its relative relationship with the existing gas station infrastructure. Our survey takes into account the infrastructure context as well as some information about the settlement type in which the participant lives in. All questions from this group are listed in Table 2. This set of questions does not only provide valuable insights into the development of existing gas station infrastructure considering settlement hierarchies, but it also measures how the familiarity with the existing transportation infrastructure impacts preferences regarding the ideal charging infrastructure.

The last set of questions, which is repeated five times to create five hypothetical scenarios, is comprised of only two questions per scenario as Fig. 2 shows. Each participant is presented with five randomly generated scenarios to assess driving range sensitivity considering key EV parameters, namely state of charge and remaining range. We again employ the technique of hidden questions concerning the range one is willing to travel to charge. In particular, that question is only presented to a participant if the answer to the previous question, about the participant's willingness to charge in the given hypothetical scenario, is affirmative.

Table 2

Driving range preferences considering settlement hierarchy.

\begin{tabular}{|c|c|c|}
\hline & QUESTION & ANSWER \\
\hline & How would you describe the place where you live? & $\begin{array}{l}\text { Village (population less than } 1,000 \text { ); } \\
\text { Town (population between } 1,000 \text { and } \\
20,000 \text { ); } \\
\text { Large town (population between } 20,000 \text { and } \\
100,000 \text { ); } \\
\text { City (population between } 100,000 \text { and } \\
300,000 \text { ); } \\
\text { Large city (population between } 300,000 \text { and } \\
1 \text { million); } \\
\text { Metropolis (population between } 1 \text { million } \\
\text { and } 3 \text { million) }\end{array}$ \\
\hline & $\begin{array}{l}\text { What is (approximately) the average distance between neighbouring GAS STATIONS in the area you live in (in } \mathrm{km} \text {, } \\
1 \mathrm{~km}=0.62 \text { miles)? }\end{array}$ & Number \\
\hline 3 & $\begin{array}{l}\text { In your opinion, what should be the maximal distance between two neighbouring GAS STATIONS in the area you live in (in } \\
\mathrm{km}, 1 \mathrm{~km}=0,62 \text { miles)? }\end{array}$ & Number \\
\hline 4 & $\begin{array}{l}\text { In your opinion, what should be the maximal distance between two neighbouring CHARGING STATIONS in the area you } \\
\text { live in (in } \mathrm{km}, 1 \mathrm{~km}=0,62 \text { miles)? }\end{array}$ & Number \\
\hline
\end{tabular}


When full, your EV can achieve maximal distance of $152 \mathrm{~km}$.

Your current state-of-charge (SoC) is $61 \%$. With that SoC you can travel the maximum of $93 \mathrm{~km}$.

Would you like to charge your EV in this circumstance during your daily city commute?
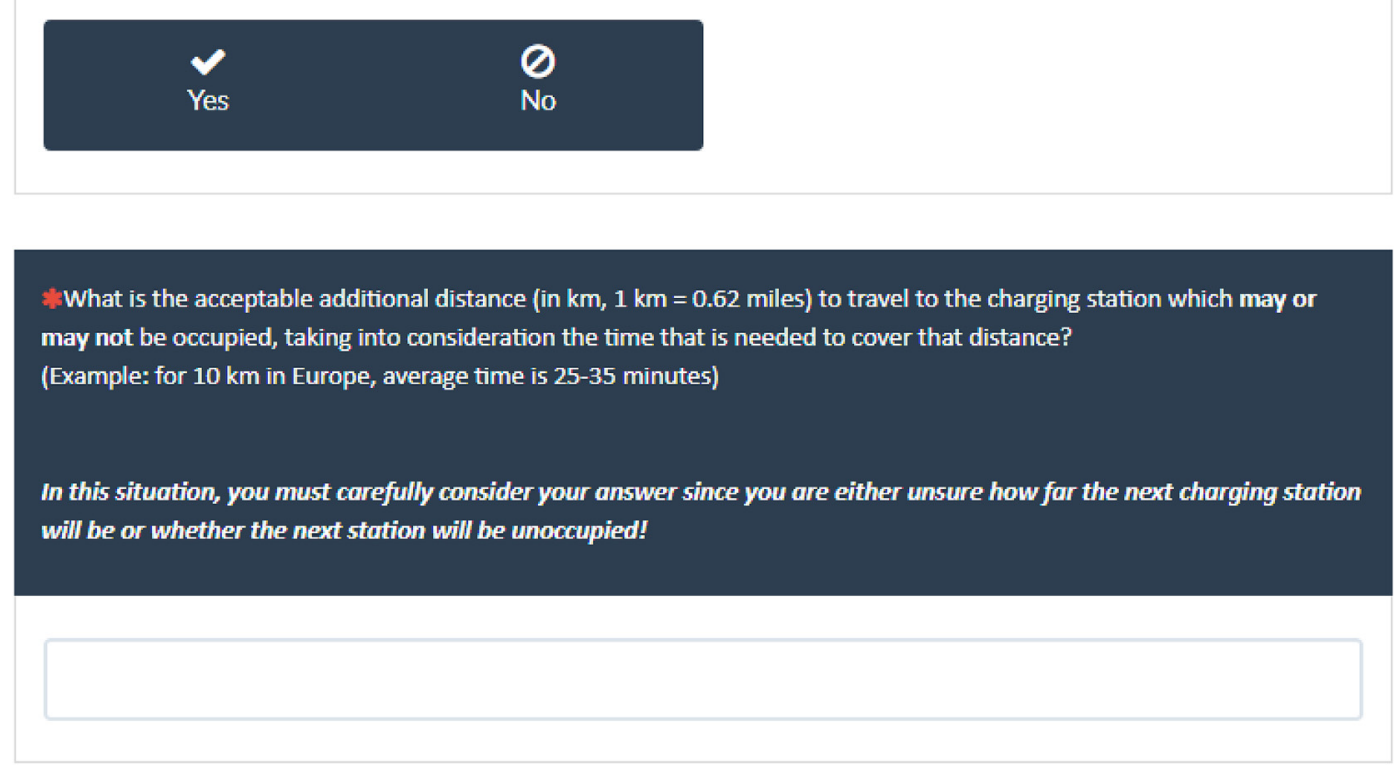

Fig. 2. Example of a randomly created scenario.

To illustrate how the scenarios were created, consider the example in Fig. 3. Note that the expressions inside square brackets are not shown to a survey participant. Instead, the expressions are replaced by values that are computed in real-time based on arbitrarily-created data relevant to each scenario. The battCap variable represents the nominal EV battery capacity, a value randomly selected between $16 \mathrm{kWh}$ and $60 \mathrm{kWh}$. This interval encodes the battery capacity of the most prevalent EVs (Ajanovic and Haas, 2018). Next, the SoC variable is used to determine the EV's state of charge. The constant value 190 is defined based on the average range of common electric vehicles per $\mathrm{kWh}$ (Ajanovic and Haas, 2018). Recall that each participant is asked to answer the same question for different five scenarios. Each scenario follows the same structure; however, the underlying parameter values change based on how the SoC variable is sampled. That said, the first scenario draws an SoC value between $5 \%$ and $100 \%$, thus representing the vast majority of realistic SoC cases. In each following scenario, the upper-bound SoC values are curtailed by $20 \%$ to encourage situations that induce range anxiety.

If the survey participant answers the first question affirmatively, i.e., $\mathrm{s} /$ he wishes to charge based on the hypothetical scenario, then the second question about the range preference is prompted as depicted in Fig. 2. This question is used to judge how a participant perceives the distance to a charging station by emphasising the

When full, your EV can achieve maximal distance of intval((battCap1 * 1000)/ 190) km. Your current state-of-charge (SoC) is SoC1 \%. With that SoC you can travel the maximum of intval $(($ battCap1 * 1000)/ 190)* (SoC1 / 100) $) \mathrm{km}$. Would you like to charge your $\mathrm{EV}$ in this circumstance during your daily city commute?

Fig. 3. Example of the First Question in Fig. 2 coded in the LimeSurvey tool. terms "additional distance", "time that is needed to cover that distance", and the fact that the charger "may or may not be occupied", as non-EV owners who are used to the high availability of traditional gas stations potentially have unrealistic expectations concerning the time needed to travel a specific distance. Also, we wanted to ensure that the participants are aware of the fact that the charging station may be out of order or occupied so they think ahead and consider the distance needed for travelling to other available charging stations without running out of electricity. As with the first question, the same survey respondent could have answered this second question multiple times; a maximum of five times, one for each affirmative answer to the first question in this set. In terms of coding, no randomization was used to create the second question, as Fig. 4 shows.

\section{Survey results}

This section describes the results of the data analysis phase concerning the demographics, desired distances between neighbouring EV charging stations, and the driving range preferences considering key EV parameters, which in turn quantity range anxiety.

What is the acceptable additional distance (in $\mathrm{km}, 1 \mathrm{~km}=0.62$ miles) to travel to the charging station which may or may not be occupied, taking into consideration the time that is needed to cover that distance? (Example: for $10 \mathrm{~km}$ in Europe, average time is 25-35 minutes) In this situation, you must carefully consider your answer since you are either unsure how far the next charging station will be or whether the next station will be unoccupied!

Fig. 4. Example of the Second Question in Fig. 2 coded in the LimeSurvey tool 


\subsection{Demographic data analysis}

The survey described in Section 3.2 was taken by 274 participants. The first group of participants, the non-EV owners, consisted of 170 participants, while the second group of participants, the EV owners, consisted of 104 participants. After the data preprocessing phase, 61 answers were removed due to one of these reasons: (i) incomplete answers, i.e., when a participant did not finish the survey; (ii) effortless answers, i.e., when a participant consistently provided the same answer, disregarding the differences in the presented scenarios (iii) inconsistent answers, e.g., some participants answered with extreme values (e.g., the lowest possible value in one scenario, and the opposite value in another); for scenarios involving battery capacities that did not differ more than a couple of $\mathrm{kWh}$, or SoCs that resulted in remaining ranges being not more than a few kilometers apart; and (iv) outliers, i.e., answers that have significant deviation from the dataset mean, which is a common procedure for noise removal (Hodge and Austin, 2004). The final number of participants considered in the data analysis is then 213 , where 134 are non-EV owners (i.e., potential EV owners) and 79 are EV owners. Table 3 shows some demographic information concerning these participants.

Achieving a substantial sample size is a challenging endeavor in our setting given the open-call and voluntary nature of our survey. A question that naturally arises is whether the sample size is enough for our purposes. We deal with that question by performing a power analysis. In particular, we perform a power analysis for an $\mathrm{F}$ test in anticipation of the fact that we shall build a multiple regression model having two predictors (see Table 5). The null hypothesis in the $\mathrm{F}$ test states that both estimated coefficients are equal to zero, whereas the alternative hypothesis states that not all estimated coefficients are equal to zero. In this setting, power (1- $\beta$ ) is influenced by the effect size $\left(f^{2}\right)$, significance level $(\alpha)$, and the sample size $(n)$. After rearranging the underlying equation, one can then determine the sample size by having a fixed effect size, significance level, and power. We rely on the traditional values of $\alpha=$ 0.05 and $\beta=0.2$. As for the effect size, given the above discussion on the challenges faced by our data collection process, we desired a sample large enough to be able to detect at least medium effects, which according to Cohen (1969) translates into an effect size of $f^{2}=0.3$. The power analysis informs us that we need a sample size of at least $n=36$ participants given the fixed parameter values. We note that both the number of non-EV owners and EV owners (respectively, 134 and 79) are considerably larger than what the power analysis suggests. In fact, with our smallest sample $(n=79)$, we can detect even smaller effect sizes, such as $f^{2}=0.13$, which in turn requires $n=78$.

The demographics of non-EV owners are consistent with those of individuals that show increased interest in electric vehicles
(Hidrue et al., 2011), in a sense that the majority of the participants are well-educated and have a stable job. Our dataset is also consistent with the fact that the survey was distributed on vehicle enthusiast forums, where the majority of the users are male. This shows as $70 \%$ of participants in this survey are also male. Since we used Facebook as one of the means for survey distribution, our pool of participants is mostly $20-40$ years old, which is consistent with the age of the majority of Facebook users. An overview of the aforementioned demographics concerning non-EV owner participants is shown in Fig. 5a. We note that the demographics of EV owners are significantly different than those of non-EV owners in terms of age and gender (Fig. 5b). In particular, most of the EV owners (85\%) are male, and more than $85 \%$ are older than 35 years old. This distribution of age can be arguably explained by the novelty of EVs on the market and the fact that they are traditionally more expensive than ICE vehicles that belong to the same category. Therefore, it is expected that one must have a stable life (secure job and income) to afford an EV.

A crucial information about the survey participants is their knowledge about EVs as well as their driving experience. EV owners, naturally, all have experience with both driving and owning EVs, while around $90 \%$ of the non-EV owner participants have at least a basic understanding of the EV concept, and more than $80 \%$ of them have a driver's license. This is important information since this study heavily relies on the distance perception between neighbouring gas stations. One should expect that the experience of driving a car increases the accuracy of that estimation.

The majority of the non-EV owner participants are from Croatia, where the charging infrastructure is rather scarce. Other participants are from 14 different countries, e.g., the United States, the United Kingdom, Norway, and other regions where EVs are more popular and, therefore, the infrastructure is better developed to accommodate one's charging needs. The aforementioned distribution of countries is beneficial for this study since, arguably, the range anxiety phenomenon is more pronounced in regions where the charging infrastructure is scarce and underdeveloped. Regarding the location of EV owners, more than $75 \%$ of the participants who own an EV are from either the United States or from the United Kingdom. This is rather expected since the EV market penetration in those countries is significantly greater than in most of the rest of the world.

\subsection{Analysis of the desired distance to a neighbouring charging station}

The analysis of the perceived and desired distances between two existing neighbouring gas stations and how they fare against the perceived and desired distances between two neighbouring charging

Table 3

Statistics concerning demographic information.

\begin{tabular}{|c|c|c|c|}
\hline Category & Subcategory & Non-EV & EV \\
\hline \multirow{3}{*}{ Working status } & & $\%$ of Participants [ $\mathrm{N}=134]$ & $\%$ of Participants [N $=79]$ \\
\hline & Employed & 67.5 & 76.5 \\
\hline & Student & 29.0 & 2.5 \\
\hline \multirow{5}{*}{ EV knowledge } & Retired & 3.5 & 20 \\
\hline & Unemployed & 0.0 & 1.0 \\
\hline & Very familiar & 45.0 & 96.0 \\
\hline & Know something & 45.0 & 4.0 \\
\hline & Heard of & 10.0 & 0.0 \\
\hline \multirow[t]{2}{*}{ Driving licence } & Have & 85.0 & 100.0 \\
\hline & Not have & 15.0 & 0.0 \\
\hline \multirow[t]{2}{*}{ Gender } & Male & 70.0 & 83.5 \\
\hline & Female & 30.0 & 16.5 \\
\hline
\end{tabular}




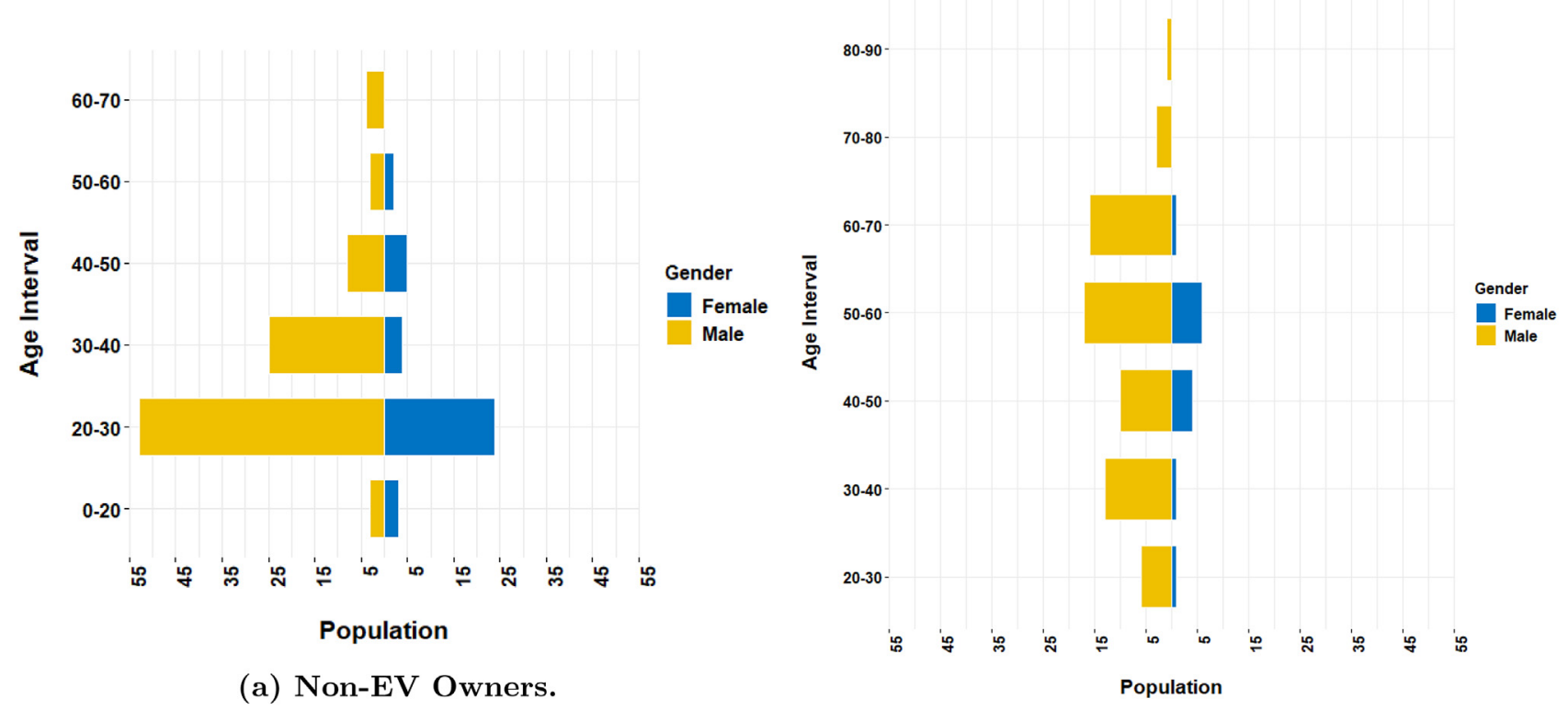

(b) EV Owners.

Fig. 5. Demographics data: Age and gender.

stations can inform decision makers about drivers' preferences when developing the underlying charging infrastructure. We assume that the maximal distance between charging/gas stations reported by the participants equates to an optimal/desired distance since such a value would fulfill their driving needs while being the least expensive option in terms of infrastructure deployment. As shown in Fig. 6a, nonEV owners' opinion regarding the traditional refueling infrastructure is that, currently, there are too many gas stations, and they are too densely distributed. In Fig. 6b, the relation between desired neighbouring gas station distances and charging station distances is compared. Evidently, potential EV owners would like the charging station infrastructure to be deployed and accessible as the traditional refueling infrastructure is. The mean distances are represented by the dashed lines in both Fig. 6a and 6b. For example, the mean desired distance between neighbouring charging stations, for non-EV owners, is around $8 \mathrm{~km}$. Fig. $6 \mathrm{c}$ and $6 \mathrm{~d}$ depict the previously described relations from the EV owner's point of view. We note that EV owners reported almost the same distance between two neighbouring gas stations as the non-EV owners. However, EV owners would prefer a closer distance between the charging stations than the non-EV owners. Arguably, the reason behind the aforementioned difference between the desired neighbouring charging station distances happens because EV owners are more knowledgeable regarding EVs and, hence, they can better assess driving ranges.

Comparison Between Real and Desired Neighbouring Gas Station Distance (Non-EV Owners). Comparison Between Desired Neighbouring Gas Station and Charging Station Distance (Non-EV Owners). Comparison Between Real and Desired Neighbouring Gas Station Distance (EV Owners). Comparison Between Desired Neighbouring Gas Station and Charging Station Distance (EV Owners).

Our data analysis suggests that more than $20 \%$ of the participants prefer the charging station infrastructure more densely distributed than what the traditional refueling infrastructure is, while about $50 \%$ of the participants would like the charging station infrastructure to be deployed as the traditional refueling infrastructure is, meaning that they are satisfied with the availability of the gas stations today. Taking into consideration the average value, our results show that two neighbouring charging stations should be $0.12 \mathrm{~km}$ less far apart than what the gas stations are. We note that we did not include a comparison between the actual and desired charging station infrastructure in this research. The reason for this lies in the fact that we surveyed participants from rather different countries, meaning that data about the actual charging infrastructure might not be uniform across different countries or even available. That is why we asked participants to estimate, based on their own empirical perception, the distance between neighbouring stations.

Recall that we asked participants about the size and type of their living area, i.e., whether they lived in a village, town, large town, city, large city, or metropolis (see Table 2). That said, there is a strong relationship between range preferences concerning neighbouring charging stations and the settlement types. In particular, participants that live in small, rural places are more prone to accepting greater distances between neighbouring charging stations than participants from larger/urban places. As can be seen in Fig. 7a and Fig. 7b, the previous statement applies to both non-EV owners and EV owners, with some minor exceptions likely due to a non-even distribution of participants across the settlement types. Another interesting finding is that non-EV owners would like the distance between two neighbouring charging stations to be less than $7.0 \mathrm{~km}$, while EV owners are satisfied with the distance of $6.8 \mathrm{~km}$ on average. Taking into account the aforementioned points, and the fact that range anxiety is often taken as the fear of running out of electricity before reaching another available charging station, the preferable distance between two neighbouring charging stations is the metric we decided to use to formally define range anxiety. However, the mean value for the preferred distance between two neighbouring charging stations is highly dependent on the settlement hierarchy. This is illustrated in Table 4, where the preferred distances for both potential and current EV owners across all settlement types are displayed.

Fig. $7 \mathrm{a}$ and $7 \mathrm{~b}$ show that there is a considerable variance in the preferred distance between neighbouring charging stations for different settlement hierarchy levels. A potential suggestion when using the values in Table 4 is to create ranges surrounding mean 


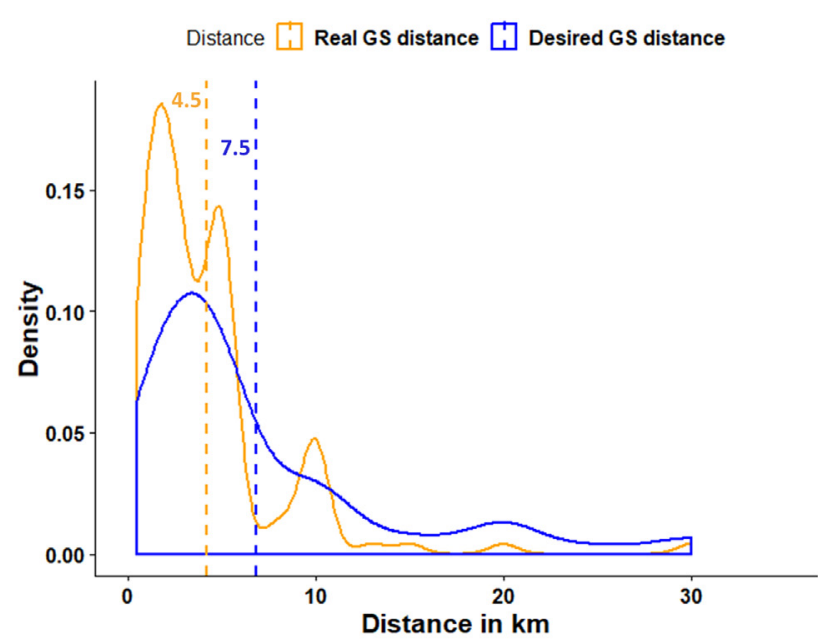

(a) Comparison Between Real and Desired Neighbouring Gas Station Distance (Non-EV Owners).

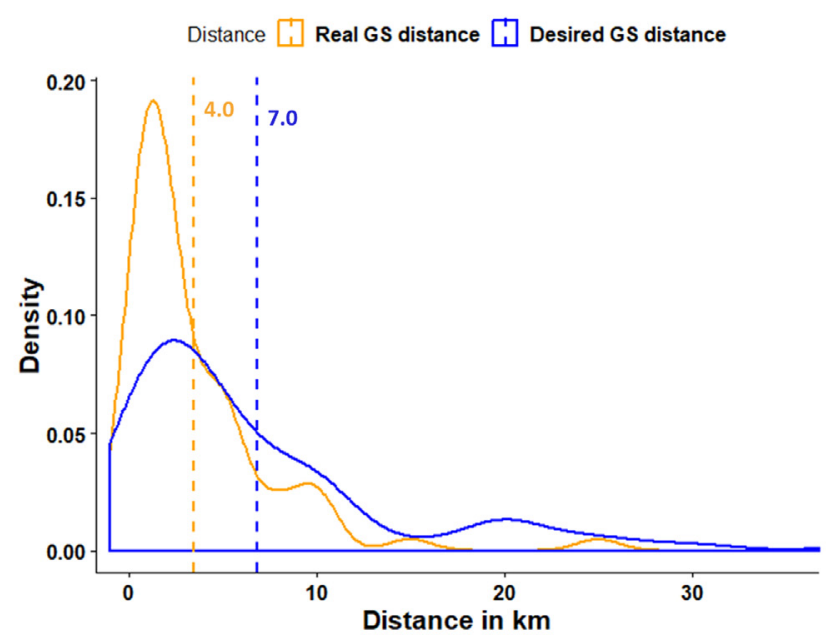

(c) Comparison Between Real and Desired Neighbouring Gas Station Distance (EV Owners).

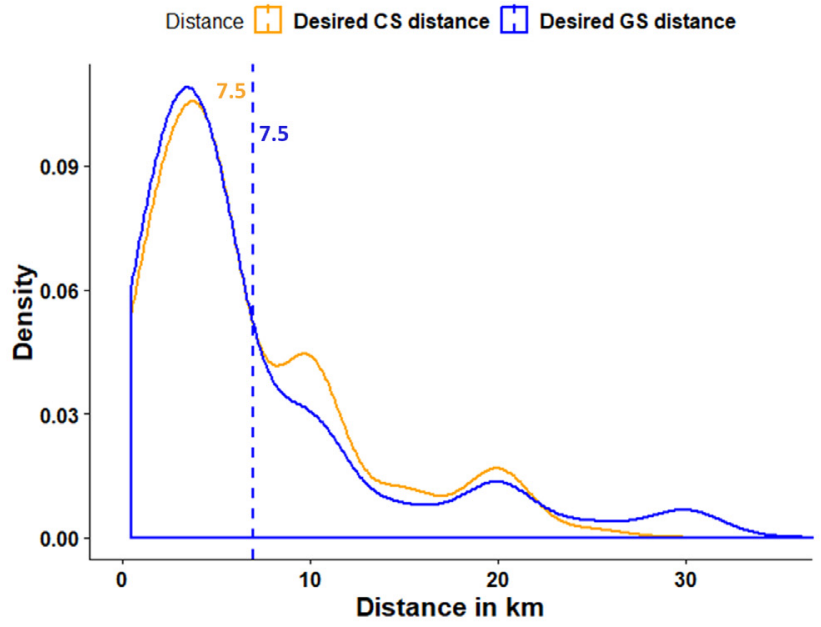

(b) Comparison Between Desired Neighbouring Gas Station and Charging Station Distance (NonEV Owners).

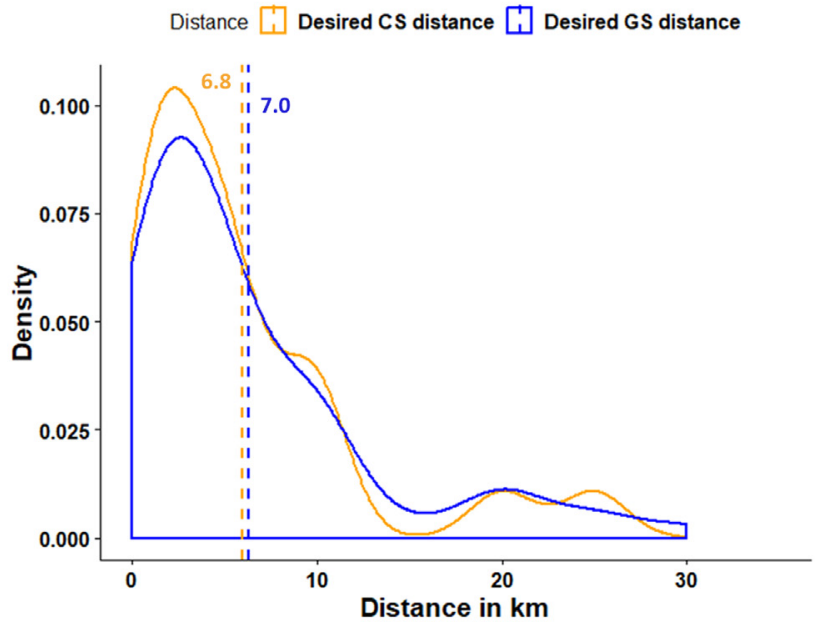

(d) Comparison Between Desired Neighbouring Gas Station and Charging Station Distance (EV Owners).

Fig. 6. Comparison between real/desired neighbouring gas/charging station distances.

values, e.g., distance preferences may vary up to one standard deviation. From the smallest to the largest settlement hierarchy level (see Table 2), the standard deviation values for EV owners are, respectively, $5.3 \mathrm{~km}, 6.6 \mathrm{~km}, 9.2 \mathrm{~km}, 3.7 \mathrm{~km}, 6.6 \mathrm{~km}$, and $1.8 \mathrm{~km}$, while these values for non-EV owners are $6.1 \mathrm{~km}, 6.6 \mathrm{~km}, 7.6 \mathrm{~km}$, $3.1 \mathrm{~km}, 4.2 \mathrm{~km}$, and $2.2 \mathrm{~km}$. Under this interpretation, the preferred distance between two charging stations for a potential EV owner from, say, a metropolis is equal to $5.0 \pm 1.8 \mathrm{~km}$. Clearly, one should restrict the lower bound of that range to zero.

The previously described findings point to the following potential conclusion: traditional refueling infrastructure is welldeveloped in larger/urban areas, which is often not the case in the smaller/rural areas and villages. Therefore, consumers from big cities might tend to be less flexible when considering the desired distances between charging stations than those who live in smaller areas. These, in turn, might be used to a smaller and more sparse refueling infrastructure.

\subsection{Range anxiety through key EV parameters}

We previously defined the key EV parameters as: (i) state of charge (SoC), i.e., the percentage of battery left; and (ii) the remaining range, i.e., the range that the vehicle can cover with the aforementioned SoC. Recall that each participant was asked to assess her/his own range anxiety, i.e., how far s/he is willing to drive to reach another available charging station. The same question was repeated up to five times to each participant, each time with a different hypothetical scenario. Therefore, the collected dataset contains up to five responses from the same participant. Those answers cannot be considered as independent as they are rather interdependent. However, they are independent from the answers reported by other participants. Taking aforementioned into account, to analyse the impact of key EV parameters on range anxiety, we used a mixed-effect model where we added a randomness term to account for the significance of the individual 


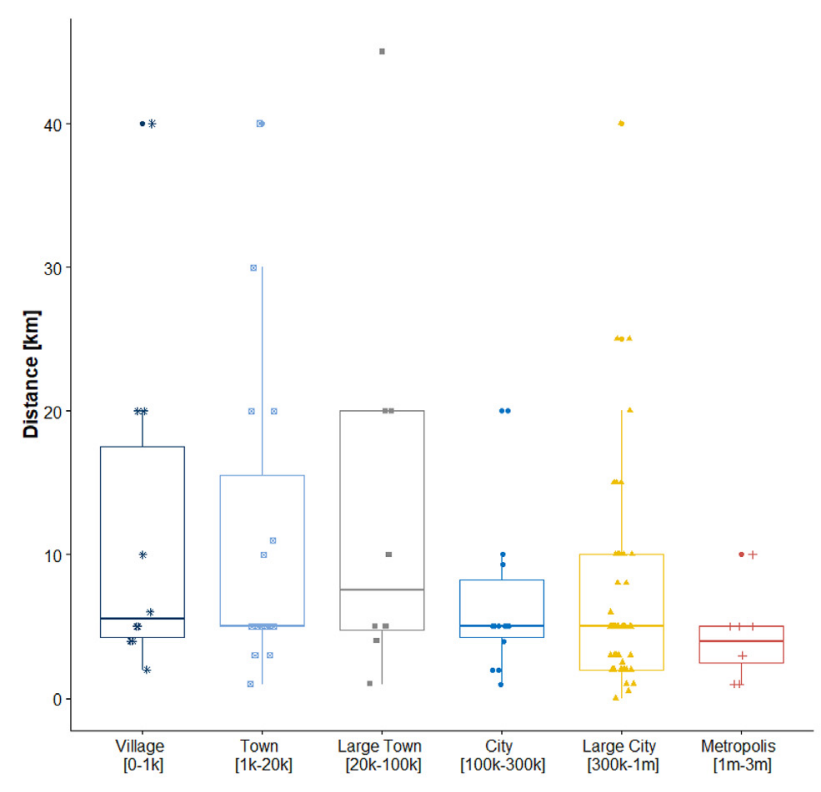

(a) Non-EV Owners

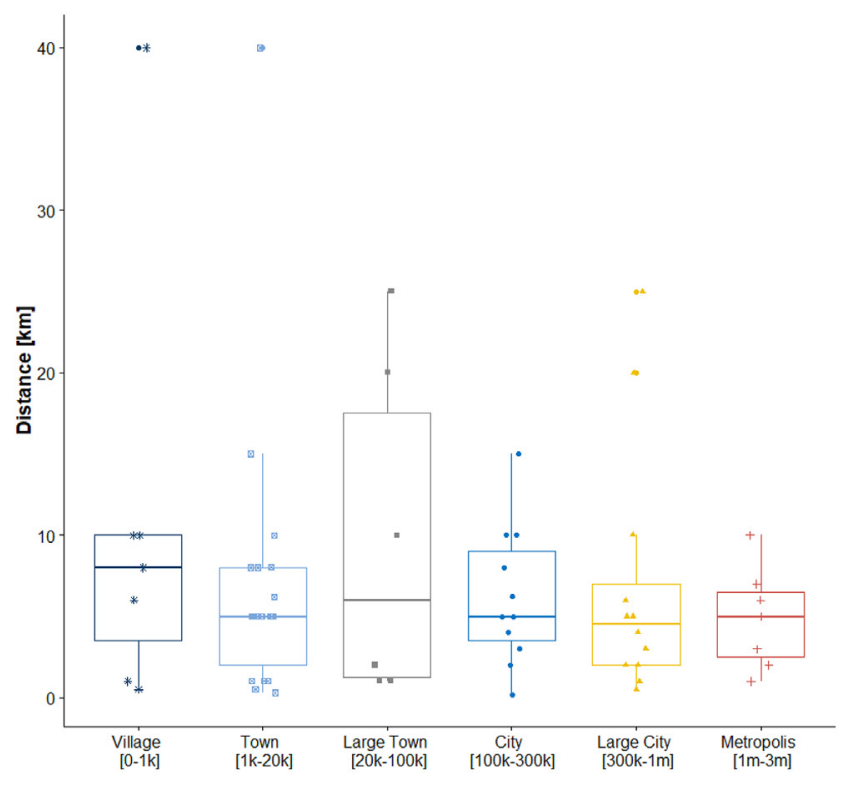

(b) EV Owners

Fig. 7. Comparison of the desired distances between neighbouring charging stations considering the settlement hierarchy.

Table 4

Preferred distances in kilometers across all settlement hierarchy levels for both EV owners and Non-EV owners.

\begin{tabular}{lll}
\hline Settlement Type & EV Owner & Non-EV Owner \\
\hline Village & 7.0 & 9.0 \\
Town & 6.8 & 6.5 \\
Large town & 9.0 & 7.0 \\
City & 6.5 & 6.6 \\
Large city & 6.5 & 6.5 \\
Metropolis & 5.0 & 6.6 \\
\hline
\end{tabular}

responses. Our mixed-effect linear regression formula is described as follows:

$$
\begin{aligned}
& \text { distance_to_travel }_{i j}=\beta_{0}+\beta_{1} * S o C_{i j} \\
& +\beta_{2} * \text { driving_range }_{i j} \\
& + \text { participant_id }{ }_{j}+\varepsilon_{i j}
\end{aligned}
$$

In Equation (1), $S o C_{i j}$ and driving_range $e_{i j}$ present the independent variables, i.e., variables with predictable impact on a variable that we want to predict. The subscript $i j$ represents the $i$-scenario faced by participant $j$. The random effect is represented by participant $_{i} i d_{j}$, an independent variable that captures variability among participants. In this model, the randomness is an interceptor that is unique for each participant, making a participant's responses mutually dependent, but also not dependent on the responses from all other participants. The above said, we built two separate regression models following Equation (1), one for each group of participants. The resulting coefficients are displayed in Table 5.

The results described in Table 5 lead us to two remarks. First, for non-EV owners, each increase in the $S o C$ unit will increase the average distance a participant is willing to travel to another available charging station by $0.08 \mathrm{~km}$ on the average, if everything else is constant. Similarly, if everything else is constant, for each increase in the unit of remaining driving_range, the distance one is willing to travel to reach another available charging station will, on average, increase by $0.22 \mathrm{~km}$. Second, for EV owners, the results point to the fact that both variables $S o C$ and the remaining driving_range have much weaker impact on the distance one is willing to travel to reach another available charging station. Specifically, if everything else is constant, increasing one unit of $\mathrm{SoC}$ results in an average increase in distance by $0.04 \mathrm{~km}$, while in the case of the remaining driving_range, that distance would decrease insignificantly.

To study the importance of the predictors, we compared our model with two variables against a baseline model using the analysis of variance (ANOVA) test. The baseline model contains only one of the variables. Our results suggest that for non-EV owners, the $\mathrm{SoC}$ variable is not significant for the prediction of the range that one is willing to travel, while, on the other hand, the driving_range variable is significant with the p-value of approximately $4 \times 10^{-10}$. For the EV owners, neither of the variables has a significant impact on the model. However, they confirm certain trends regarding the key EV parameters, i.e., participants are willing to travel further the more SoC they have. To ensure that there is no multicollinearity between our independent variables, we calculated the variance inflation factor (VIF), which is equal to 2.0, thus leading to the conclusion that the standard error is only 1.4 times larger than if a predictor variable ( $\mathrm{SoC}$ ) had 0 correlation with the other predictor variable (remaining range). In terms of goodnessof-fit, we calculate two r-squared measures based on the work by Nakagawa and Schielzeth (2013). In particular, marginal R-squared provides the variance explained only by fixed effects, while conditional R-squared provides the variance explained by the entire model, i.e., by both fixed and random effects. Both conditional rsquared values show that our models fit well our data.

The presented range anxiety model is illustrated in the next example considering non-EV owners. Assume that a person considers purchasing a BMWi with the battery of $50 \mathrm{kWh}$ capacity (i.e., the nominal distance that such EV can cover is around $210 \mathrm{~km}$ ). If that individual would find himself/herself in a hypothetical scenario having the SoC at the $20 \%$ level (i.e., the remaining range is $42 \mathrm{~km}$ ), s/he would then agree to travel for $8.4 \mathrm{~km}$ to reach a charging station in order to charge.

As Fig. 8a shows, non-EV owners care less about the state of charge than the remaining range that they can cover. This 
Table 5

Description of the obtained mixed-effect models.

\begin{tabular}{|c|c|c|c|c|c|}
\hline Non-EV Owners & & & EV Owners & & \\
\hline \multicolumn{6}{|l|}{ Random effects } \\
\hline Groups & Variance & Std. Dev. & Groups & Variance & Std. Dev. \\
\hline participant_id & 178.5 & 13.36 & participant_id & 31.70 & 5.63 \\
\hline Residual & 218.8 & 14.79 & Residual & 17.18 & 4.14 \\
\hline \multicolumn{6}{|l|}{ Fixed effects } \\
\hline Coefficients & Estimate & p-value & Coefficients & Estimate & p-value \\
\hline Intercept $\left(\beta_{0}\right)$ & -1.84 & 0.35 & Intercept $\left(\beta_{0}\right)$ & 6.08 & $<0.01$ \\
\hline SoC $\left(\beta_{1}\right)$ & 0.08 & 0.34 & $\operatorname{SoC}\left(\beta_{1}\right)$ & 0.04 & 0.08 \\
\hline driving_range $\left(\beta_{2}\right)$ & 0.22 & $<0.01$ & driving_range $\left(\beta_{2}\right)$ & -0.002 & 0.76 \\
\hline \multicolumn{6}{|c|}{ Goodness-of-Fit Measures } \\
\hline Marginal $R^{2}$ & 0.23 & & Marginal $R^{2}$ & 0.01 & \\
\hline Conditional $R^{2}$ & 0.58 & & Conditional $R^{2}$ & 0.65 & \\
\hline
\end{tabular}

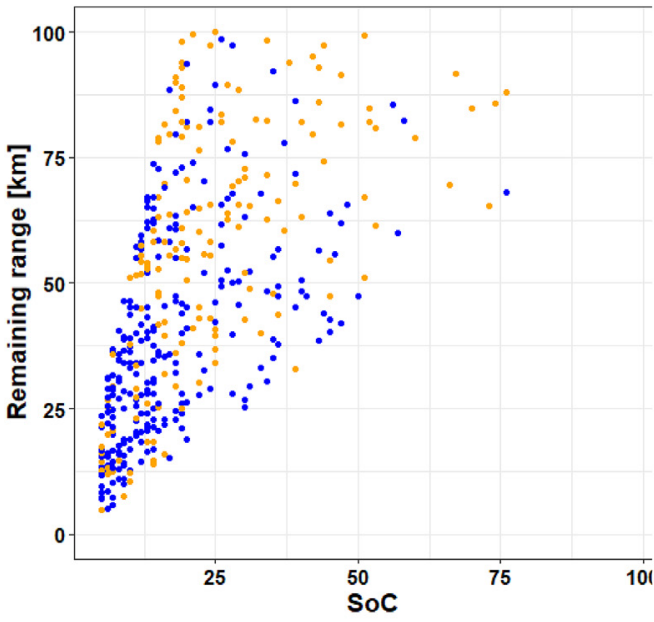

(a) Non-EV owners

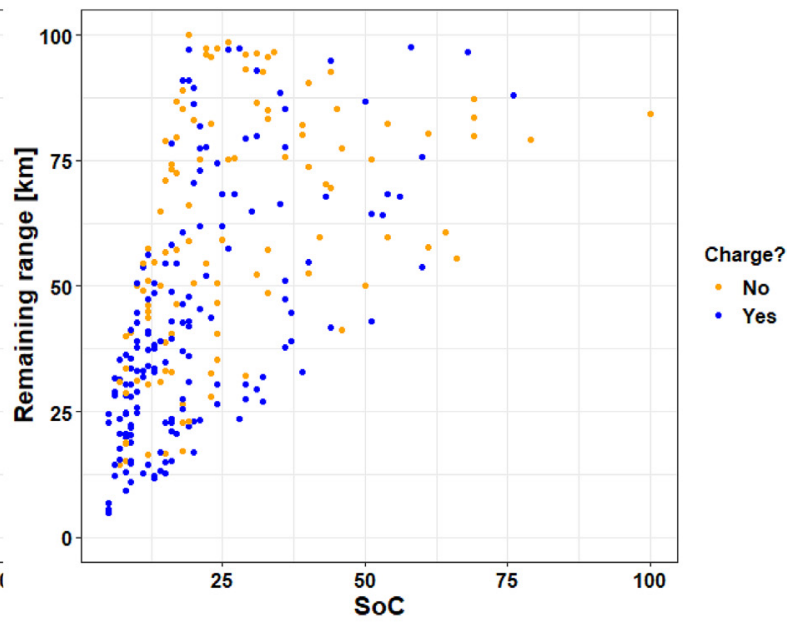

(b) EV owners

Fig. 8. Comparison of the willingness to charge considering SoC and remaining range.

conclusion is expected since two vehicles with the same SoC can cover significantly different distance, e.g., a Nisan Leaf with $30 \mathrm{kWh}$ battery capacity and with $15 \%$ SoC can cover up to $24 \mathrm{~km}$, while Tesla Model S with $95 \mathrm{kWh}$ on the same SoC can cover more than $70 \mathrm{~km}$. EV owners have different perspective, as can be seen in Fig. 8 b, where those that are willing to charge are more scarcely distributed between 0 and $50 \%$ SoC, as well as between 0 and $100 \mathrm{~km}$ of the remaining range. The main reason behind this phenomenon is the fact that some EV owners own a private charging station (e.g., can charge at home), and others have accessible chargers near their workplaces, and for that reason they do not react as expected to different values of SoC and the remaining range. This is discussed in more details in the next section.

\section{Discussion}

Our results suggest that non-EV owners are more affected by the key EV parameters than the EV owners when determining the distance they are prepared to drive in order to find an available charging station. This finding is also aligned with previous studies conducted by other researchers presented in Section 2. Furthermore, the remaining driving range has a stronger influence on range anxiety than the SoC for non-EV owners. This influence can be further enhanced by advertisements educating potential EV owners on the nominal range an EV can cover and the distances one is approximately covering in a week drive, since modern EVs have the battery capacity large enough to satisfy an average customer's day-to-day commuting needs. However, even if the remaining range variable is more significant than the $\mathrm{SoC}$, SoC still gives us important insights into the range anxiety phenomenon in the context of non-EV owners. Namely, most of the non-EV owners are more inclined to charge when SoC drops below 15\%, a level at which most of the EVs prompt a warning about low battery capacity.

How the key EV variables impact EV owners' thinking about charging is still undetermined. The reason behind this is the fact that this survey is universal for both non-EV owners and EV owners, i.e., not specifically designed with EV owners in mind. EV owners have experience in driving an EV and they have different habits when it comes to charging. This fact is reflected on the comments left by some respondents from EV specialized forums. The majority of these comments were addressing the fact that early adopters tend to own a private charger and that they never let the SoC fall below $30 \%$. Therefore, some of the EV owners are willing to charge whenever they have an opportunity, since that is what they are used to do as they plug in their vehicle whenever they are at home. In fact, the majority of the EV owners that participated in our research are enthusiasts - a consequence of targeting the audience through the EV-focused forums - that own a private charger. Moreover, the majority of the EV owners are early adopters that live in smaller settlements, often having a population below 300,000. Therefore, they are accustomed to drive further to reach a gas station and they would not mind the same in the case of the charging station. 
One of the greatest challenges faced by our research regards participants' perception of the distance. Some participants reported that the acceptable distance to travel to the nearest charging station is more than $50 \mathrm{~km}$, while a minority of the participants stated that they are willing to travel for the distance that equals their whole available driving range to find a charging station, disregarding the fact that the same could be out of order or occupied. This attitude is not a surprise as some of the survey respondents may be more prone to taking risks than the others. To solve the aforementioned issue, answers that greatly differ from the dataset mean value were removed in the process of outlier detection and removal, as explained in Section 3. The outlier removal process was performed with great attention to settlement hierarchy, since the majority of non-EV owners participants were from larger settlements and they dictate the mean of the dataset, while the participants from smaller settlements are expected to be willing to travel for greater distances.

An interesting observation is that, despite the aforementioned differences between non-EV owners and EV owners regarding the charging habits and settlement types, both groups of participants provided a similar approximation of the distances between existing neighbouring gas stations. Furthermore, both participant groups also reported similar desired distances between two neighbouring charging stations, leading us to the conclusion that the significant underdevelopment of the charging station infrastructure is still the main cause of the range anxiety phenomenon.

It is fair to acknowledge the limitations of our research, some of which are related with the characteristics of the respondents who participated in our survey, while others are related to the statistical approach used to analyse and interpret the survey results. The most important limitation arising from the survey respondents perspective is the fact that the majority of survey participants who are EV owners are located in the United States or the United Kingdom, while the majority of non-EV owners are from Croatia. Although we, at least partially, captured the information about the survey respondents' location context through their settlement sizes, a follow up survey with more comprehensive respondent pool would enhance the generalizability of our conclusions by fully removing biases rooted in different drivers' cultures and characteristics due to their geographical location. The most important limitations arising from the statistical approach we used are: (i) the existence of an almost linear relationship between SoC and the driving range; and (ii) the usage of a linear model to explain range anxiety based on SoC and the driving range, which resulted in some statistically insignificant results. Regarding the former limitation, a deeper statistical analysis of the collected survey responses provided grounding for having both SoC and driving range in the model, what can potentially be explained by how (potential) EV owners interpret those two parameters. However, again, a more comprehensive study focused specifically on such the interpretation of such parameters should be done to confirm our conclusion. Regarding the latter limitation, it would be beneficial to extend statistical modeling beyond linear to explore whether more complex modelling approaches would better capture the relationships between the underlying variables.

\section{Conclusion}

The results presented in this paper provide insights into how far apart neighbouring charging stations should be in order to mitigate range anxiety. Industry and governments can use our findings to popularize EVs, resulting in more drivers purchasing EVs as opposed to traditional ICE vehicles. This can also be of great value for researchers studying the management of charging station infrastructures, as the desired span between neighbouring charging stations needs to be taken into consideration when creating relevant scientific models.

The analysis of data gathered from respondents to the specially created survey enabled us to answer both overarching research questions presented in Section 1. In particular, both EV owners as well as non-EV owners share the opinion that the gas station infrastructure is overdeveloped, i.e., neighbouring gas stations can be further apart than they currently are. Moreover, both participant groups choose the average desired span between neighbouring charging stations to be around $7 \mathrm{~km}$, which corresponds with the preferred distance between neighbouring gas stations. Another important conclusion comes from grouping survey respondents based on settlement types: survey respondents from smaller settlements are satisfied with longer spans between neighbouring charging stations when compared to respondents from larger settlements, which also somehow reflects the current topology of the gas infrastructure network. Regarding the impact of key range anxiety variables on non-EV owners, we identified that SoC has more influence compared to remaining range on when non-EV owners decide about whether to charge, while on the other hand remaining range has more impact when deciding about the distance a non-EV owner wants to travel for reaching another charging station. EV owners show slightly similar trends as the non-EV owners, e.g., they are more prone not to charge when their EV's SoC is higher. However, EV owners are less sensitive about the key EV parameters since they have a real-life experience with EVs. This also leads to the conclusion that the experience of owning an EV greatly influences range anxiety. Overall, we argue that these results are of great importance to the green transportation area since they can be used in various decision support systems aimed at charging station deployment. For example, if the results presented in this paper are taken into consideration when deploying new charging stations, range anxiety can potentially be lowered and, consequently, this can influence a potential EV owner's decision to buy an electric vehicle.

For future work, we plan to customize the survey based on the feedback that we received from the EV owners, e.g., it is important to know whether someone owns a private charger since then there is no need for the access to the public charging infrastructure. This piece of information can greatly influence their responses considering the preferred distances, as well as their perception of the key EV parameters. Another interesting aspect for understanding the range anxiety phenomenon that we identified in this paper is the influence of settlement type a (potential) EV owner is living in. In the future, we plan to focus on this aspect of the research by targeting a sufficient number of respondents in each of the settlement hierarchy type. Finally, we plan to mitigate some of the identified limitations of this paper in a follow-up research, including the distribution of the extended survey among respondents form a more geographically balanced base, i.e., have a more even distribution of participants from more countries, instead of primarily from Croatia, the United Kingdom, and the United States, as well as use more complex statistical approaches for interpreting the collected data in order to achieve more statistically significant results.

\section{CRediT authorship contribution statement}

Dario Pevec: Conceptualization, Methodology, Software, Validation, Investigation, Writing - original draft, Visualization. Jurica Babic: Conceptualization, Methodology, Writing - original draft, Writing - review \& editing. Arthur Carvalho: Conceptualization, Methodology, Software, Formal analysis, Writing - review \& editing. Yashar Ghiassi-Farrokhfal: Investigation, Writing - original draft, Writing - review \& editing. Wolfgang Ketter: Investigation, 
Writing - original draft, Writing - review \& editing. Vedran Podobnik: Conceptualization, Methodology, Writing - original draft, Writing - review \& editing, Supervision.

\section{Declaration of competing interest}

The authors declare that they have no known competing financial interests or personal relationships that could have appeared to influence the work reported in this paper.

\section{References}

Adnan, N., Nordin, S.M., Rahman, I., Vasant, P.M., Noor, A., 2017. A comprehensive review on theoretical framework-based electric vehicle consumer adoption research. Int. J. Energy Res. 41, 317-335.

Agency, E.E., 2019. Progress of eu transport sector towards its environment and climate objectives. Available at. https://www.eea.europa.eu/themes/transport/ term/term-briefing-2018.

Ajanovic, A., Haas, R., 2018. Electric vehicles: solution or new problem? Environ. Dev. Sustain. 20, 7-22.

Akakpo, A., Gyasi, E.A., Oduro, B., Akpabot, S., 2019. Foresight, organization policies and management strategies in electric vehicle technology advances at tesla. In: Futures Thinking and Organizational Policy. Springer, pp. 57-69.

Babic, J., Carvalho, A., Ketter, W., Podobnik, V., 2017. A data-driven approach to manage charging infrastructure for electric vehicles in parking lots. Available at. https://papers.ssrn.com/sol3/papers.cfm?abstract_id=3035913.

Bjerkan, K.Y., Nørbech, T.E., Nordtømme, M.E., 2016. Incentives for promoting battery electric vehicle (bev) adoption in Norway. Transport. Res. Transport Environ. 43, 169-180.

Bonges III, H.A., Lusk, A.C., 2016. Addressing electric vehicle (ev) sales and range anxiety through parking layout, policy and regulation. Transport. Res. Pol. Pract. 83, 63-73.

Bunsen, T., Cazzola, P., Gorner, M., Paoli, L., Scheffer, S., Schuitmaker, R., Tattini, J., Teter, J., 2018. Global Ev Outlook 2018: towards Cross-Modal Electrification. International Energy Agency.

Cohen, J., 1969. Statistical Power Analysis for the Behavioral Sciences. Academic press.

Couper, M.P., Traugott, M.W., Lamias, M.J., 2001. Web survey design and administration. Publ. Opin. O, 65, 230-253.

Dorcec, L., Pevec, D., Vdovic, H., Babic, J., Podobnik, V., 2019. How do people value electric vehicle charging service? a gamified survey approach. J. Clean. Prod. 210, 887-897.

Franke, T., Krems, J.F., 2013. What drives range preferences in electric vehicle users? Transport Pol. 30, 56-62.

Hidrue, M.K., Parsons, G.R., Kempton, W., Gardner, M.P., 2011. Willingness to pay for electric vehicles and their attributes. Resour. Energy Econ. 33, 686-705.

Higgins, A., Paevere, P., Gardner, J., Quezada, G., 2012. Combining choice modelling and multi-criteria analysis for technology diffusion: an application to the uptake of electric vehicles. Technol. Forecast. Soc. Change 79, 1399-1412.

Hirschman, A.O., 1991. The Rhetoric of Reaction. Harvard University Press.

Hodge, V., Austin, J., 2004. A survey of outlier detection methodologies. Artif. Intell. Rev. 22, 85-126.

Jensen, A.F., Cherchi, E., Mabit, S.L., Ortúzar, J.d.D., 2016. Predicting the potential market for electric vehicles. Transport. Sci.

Jung, M.F., Sirkin, D., Gür, T.M., Steinert, M., 2015. Displayed uncertainty improves driving experience and behavior: the case of range anxiety in an electric car. In: Proceedings of the 33rd Annual ACM Conference on Human Factors in
Computing Systems. ACM, pp. 2201-2210.

Kahlen, M.T. Ketter, W. van Dalen, J., 2018. Electric vehicle virtual power plant dilemma: grid balancing versus customer mobility. Prod. Oper. Manag. 27, 2054-2070.

Ketter, W., Peters, M., Collins, J., Gupta, A., 2016. A multiagent competitive gaming platform to address societal challenges. MIS O. 40, 447-460.

King, C., Griggs, W., Wirth, F., Quinn, K., Shorten, R., 2015. Alleviating a form of electric vehicle range anxiety through on-demand vehicle access. Int. J. Contr. 88, 717-728.

Ko, W., Hahn, T.K., 2013. Analysis of consumer preferences for electric vehicles. IEEE Trans. Smart Grid 4, 437-442.

Lebeau, K., Van Mierlo, J., Lebeau, P., Mairesse, O., Macharis, C., 2012. The market potential for plug-in hybrid and battery electric vehicles in flanders: a choicebased conjoint analysis. Transport. Res. Transport Environ. 17, 592-597.

Nakagawa, S., Schielzeth, H., 2013. A general and simple method for obtaining r2 from generalized linear mixed-effects models. Methods Ecol. Evol. 4, 133-142.

Neubauer, J., Wood, E., 2014. The impact of range anxiety and home, workplace, and public charging infrastructure on simulated battery electric vehicle lifetime utility. J. Power Sources 257, 12-20.

Noel, L., de Rubens, G.Z., Sovacool, B.K., Kester, J., 2019. Fear and loathing of electric vehicles: the reactionary rhetoric of range anxiety. Energy Res. Soc. Sci. 48, 96-107.

Paavola, J., 2017. Health impacts of climate change and health and social inequalities in the UK. Environ. Health 16, 113.

Pevec, D., Babic, J., Carvalho, A., Ghiassi-Farrokhfal, Y., Ketter, W., Podobnik, V., 2019 Electric vehicle range anxiety: an obstacle for the personal transportation ( $\mathrm{r}$ ) evolution?. In: 2019 4th International Conference on Smart and Sustainable Technologies (SpliTech), IEEE, pp. 1-8.

Pevec, D., Babic, J., Kayser, M.A., Carvalho, A., Ghiassi-Farrokhfal, Y., Podobnik, V., 2018. A data-driven statistical approach for extending electric vehicle charging infrastructure. Int. J. Energy Res. 42, 3102-3120.

Plötz, P., Schneider, U., Globisch, J., Dütschke, E., 2014. Who will buy electric vehicles? identifying early adopters in Germany. Transport. Res. Pol. Pract. 67 96-109.

Quinn, R., Moseman-Valtierra, S., Kroeger, K., Martin, R., Abdul-Aziz, O., Ishtiaq, K. Brannon, E., Egan, K., Tang, J., 2016. The coastal squeeze: rising seas and upland plant invasions differentially affect vertical exchange of greenhouse gases. In: American Geophysical Union, vol. 2016. Ocean Sciences Meeting abstract\# EC14B-0974.

Rauh, N., Franke, T., Krems, J.F., 2015. Understanding the impact of electric vehicle driving experience on range anxiety. Hum. Factors 57, 177-187.

Statista, 2018. Electric vehicles worldwide. Available at. https://www.statista.com/ study/11578/electric-vehicles-statista-dossier/.

Valogianni, K., Ketter, W., 2016. Effective demand response for smart grids: evidence from a real-world pilot. Decis. Support Syst. 91, 48-66.

Valogianni, K., Ketter, W., Collins, J., Zhdanov, D., 2018. Facilitating a sustainable electric vehicle transition through consumer utility driven pricing. In: 2018 International Conference on Information Systems, San Francisco, California, United States.

Valogianni, K., Ketter, W., Collins, J., Zhdanov, D., 2020. Sustainable electric vehicle charging using adaptive pricing. Prod. Oper. Manag.

Valogianni, K., Ketter, W., De Weerdt, M., Collins, J., 2012. Modeling electric vehicle customers and learning a heuristic charging behaviour. In: Conference in Information Systems and Technology.

Van Fan, Y., Perry, S., Klemeš, J.J., Lee, C.T., 2018. A review on air emissions assessment: Transportation. J. Clean. Prod. 194, 673-684.

Wirth, R., Hipp, J., 2000. Crisp-dm: towards a standard process model for data mining. In: Proceedings of the 4th International Conference on the Practical Applications of Knowledge Discovery and Data Mining, Citeseer, pp. 29-39. 